EPJ Web of Conferences 106, 03004 (2016)

DOI: $10.1051 /$ epjconf/201610603004

(C) Owned by the authors, published by EDP Sciences, 2016

\title{
Radiation Damage Assessment in the Reactor Pressure Vessel of the Integral Inherently Safe Light Water Reactor (I'S-LWR)
}

\author{
Timothy Flaspoehler ${ }^{\mathrm{a}}$ and Bojan Petrovic \\ Georgia Institute of Technology, Nuclear and Radiological Engineering, 770 State St., Atlanta \\ GA 30332-0745, USA
}

\begin{abstract}
One of the major limiting factors to nuclear reactors lifetime is the radiationinduced material damage in the Reactor Pressure Vessel (RPV). While older reactors were designed assuming a 40-year operating lifetime, new reactor designs are expected to have lifetimes up to 100 years. For safe operation, the integrity of the RPV must be ensured against significant material property changes. In this work, typical neutron damage indicators are calculated in the RPV of the $I^{2}$ S-LWR (Integral Inherently Safe LWR) Power Plant, including DPA (displacements per atom) and fast neutron fluence $(>1 \mathrm{MeV}$ and $>0.1 \mathrm{MeV}$ ). $\mathrm{I}^{2} \mathrm{~S}-\mathrm{LWR}$ is a PWR of integral design, which means that its wider downcomer provides additional shielding to the vessel. However, its higher core power density and longer lifetime may offset this advantage. In order to accurately represent the neutron environment for RPV damage assessment, a detailed model based on the preliminary design specifications of the $\mathrm{I}^{2}$ S-LWR was developed to be used in the MAVRIC (Monaco with Automated Variance Reduction using Importance Calculations) sequence of the Scale6.1 code package. MAVRIC uses the CADIS (Consistent Adjoint-Driven Importance Sampling) methodology to bias a fixed-source MC (Monte Carlo) simulation. To establish the upper limit of a bounding envelope, a flat-source distribution was used. For the low limit, a centerpeaked source was generated using the KENO-VI criticality sequence assuming uniform fresh fuel core. Results based on the preliminary $\mathrm{I}^{2} S$-LWR model show that DPA rates and fast fluence rates are conservatively $75 \%$ lower than in typical PWRs being operated currently in the US.
\end{abstract}

\section{Introduction}

The purpose of this study was to better understand and quantify the fast fluence and DPA rates [1] in the RPV of the $\mathrm{I}^{2} \mathrm{~S}-\mathrm{LWR}$ (Integral Inherently Safe LWR). The $\mathrm{I}^{2} \mathrm{~S}-\mathrm{LWR}$ seeks to combine safety aspects of SMR (Small Modular Reactor) designs with economic benefits of large LWRs [2, 3]. By integrating primary components within the RPV (Reactor Pressure Vessel), accident scenarios such as LB-LOCAs (Large Break Loss of Coolant Accidents) are avoided by not being possible within the design. Safety is further increased by using a fuel-cladding system based on uranium silicide fuel and FeCrAl-type (such

\footnotetext{
${ }^{\text {a }}$ Corresponding author: timothy.flaspoehler@gatech.edu
}

This is an Open Access article distributed under the terms of the Creative Commons Attribution License 2.0, which permits unrestricted use, distribution, and reproduction in any medium, provided the original work is properly cited. 
as APMT) advanced steel cladding. Furthermore, extra components such as a steel reflector and larger downcomer region provide additional shielding to the RPV.

Traditionally, the radiation-induced material change within the RPV is considered to be one of the main limitations regarding NPP (nuclear power plant) lifetime assessment. Neutron-induced changes to material properties in the RPV, specifically at its welds close to the core midplane, lead to a higher susceptibility to pressurized thermal shock (PTS) during transient events. In the past, lower leakage core loading patterns were implemented by replacing fresh fuel assemblies on the periphery with burnt ones in order to reduce the risk of PTS. The change in loading pattern improves the neutron economy but may increase radial peaking. The integral $\mathrm{I}^{2} \mathrm{~S}$-LWR design with its wider downcomer provides additional shielding and flux attenuation compared to loop PWRs [4]. However, its higher volumetric core power density and longer lifetime may offset this advantage. Moreover, a range of refuelling strategies is considered that will ultimately impact the RPV fluence and introduce uncertainty over the envisioned 100 -year lifetime. It has been estimated that the wider downcomer will not only mitigate these opposing effects but also provide a margin to allow flexibility in future operation. The purpose of this study is to confirm and quantify this margin under most conservative assumptions.

ASTM standard E706 [5] and NRC regulatory guide 1.190 [6] for reactor dosimetry calculations require a fixed source to be defined based on the time-averaged fission rate taking into account the number of neutrons generated and spectrum created from different isotopics. A fission source distribution and spectrum over the full lifetime of a reactor [7] can be difficult to quantify, since it requires full-core depletion through multiple batches moving towards an equilibrium fuel cycle and predicting the future reloading strategy for the 100-year operating lifetime. Since the goal of this study was to perform a scaling analysis of neutron fluence to the RPV with respect to different parameters, a more simplified approach was used which would envelope maximum and minimum bounds. By creating an envelope possibly different fuel cycle scenarios could be designed without the limitation of PTS events at weld locations in the RPV [8-10].

Steels exposed to enough neutron fluence experience radiation damage that can degrade certain material properties [11]. Of specific interest to nuclear power plants is the effects of embrittlement on the RPV. Embrittlement measures how much energy a material can absorb without rupturing. The important parameter relating to neutron embrittlement is the RTNDT (Reference Temperature for Nil Ductility Transition). At lower temperatures steels are more brittle in nature (and fracture more easily), but become more ductile (and less susceptible to rupture) as the temperature rises above the RTNDT. High enough neutron fluences lower this transition temperature, which makes the vessel more susceptible to rupture during transients, a process known as a PTS event. NUREG 1.99 [12] provides details on calculating the change in RTNDT with respect to chemical composition (copper and nickel) and fast neutron fluence. The change in RTNDT, or $\triangle$ RTNDT, from neutron fluence begins to be significant around a fast neutron fluence of $2\left(10^{19}\right) \mathrm{n} / \mathrm{cm}^{2}$ for welds with high $\mathrm{Cu}$ and $\mathrm{Ni}$ content, and we use this value as the conservative upper allowed limit at the assumed 100-year lifetime. Additionally, the regulatory guide suggests that the radial distribution through the RPV of the $\triangle$ RTNDT from neutron fluence is better approximated by the accumulated DPA distribution which attenuates more in steels than the fast fluence.

In this study two different fixed source descriptions will be investigated in order to develop an envelope for fast fluence to the RPV. The simplest, most basic source is a spatially flat source with a Uranium-235 fission spectrum, which effectively results in higher leakage. As mentioned above, older core loading patterns were closer to a flat-source approximation which has the benefit of lower radial peaking, but increases the source at the core periphery leading to a higher fluence to the RPV. The second center-peaked source distribution results from considering a uniform (in this case all fresh fuel) core composition in an MC (Monte Carlo) eigenvalue calculation. No control rods or burnable absorbers have been used in this model, which leads to high peaking in the core center and a low source at the periphery which underestimates fluence to the RPV. In this manner an envelope for fast fluence is defined. 


\section{$15^{\text {th }}$ ISRD}

\section{I'S-LWR}

\subsection{Fuel Cycle and Core Description}

The $\mathrm{I}^{2} \mathrm{~S}$-LWR design increases volumetric power density by $20-40 \%$ from typical 2-loop cores. Improving safety while at the same time increasing the power density requires using novel materials for both fuel and cladding. Uranium silicide fuel replaces uranium oxide fuel to allow a higher heavy metal density. Additionally, an inner annulus in silicide pellets provides room for inward swelling of the fuel which is expected to swell more than oxide fuel. An advanced SS (Stainless Steel) replaces zirconium in the cladding as well as spacer grids. The combined result of the novel fuel and cladding system is a more accident tolerant fuel, with lower temperature in normal operation and reduced oxidation rate in loss of coolant accident scenarios. Stainless steel cladding has a proven history of use in LWRs in the past, but was replaced due to its neutron penalty with respect to Zircaloy. The penalty ultimately leads to higher fuel enrichment costs, but is necessary for the integral configuration to attain goals with respect to increased safety performance. Economically, the integral configuration is expected to offset the increased fuel cost by allowing for lower construction costs on the front end. Additionally, increasing the fuel assembly lattice structure to a $19 \times 19$ configuration further supports the higher power density and heat removal.

The annular $\mathrm{U}_{3} \mathrm{Si}_{2}$ fuel pellets are used with a heavy-metal density assumed to be $96.5 \%$ of the theoretical density of $12.2 \mathrm{~g} / \mathrm{cc}$. The active fuel rods have a height of $365.76 \mathrm{~cm}$ with inner and outer radii of 0.127 and $0.4015 \mathrm{~cm}$ respectively. The gap (helium) and cladding (SS) thicknesses are $0.015 \mathrm{~cm}$ and $0.041 \mathrm{~cm}$ respectively. The fuel rod pitch is $1.21 \mathrm{~cm}$ with an assembly pitch of $23.1 \mathrm{~cm}$. In total 121 $19 \times 19$ fuel assemblies make the core (Fig. 1).

The core is radially enclosed by a neutron steel reflector made of SS-304 with an outer radius of $165 \mathrm{~cm}$. Due to the proximity to the core, cooling channels will be provided within the steel reflector. The detailed design has not been completed at this time. To assess the impact, the reflector will be approximated in this study by three different steel and water volume ratios $(100 \% / 0 \%, 90 \% / 10 \%$, $70 \% / 30 \%$ ). Surrounding the reflector is a downcomer region that has a thickness of $70 \mathrm{~cm}$ and an outer radius of $235 \mathrm{~cm}$. The large downcomer region provides more shielding than in a typical PWR which leads to a reduction in the neutron fluence to the RPV. The RPV is composed of low-carbon steel with stainless steel clad (not included in model) and has a thickness of $27.5 \mathrm{~cm}$ and inner and outer radii of $235 \mathrm{~cm}$ and $262.5 \mathrm{~cm}$ respectively.

\section{Fixed-Source Distribution}

\subsection{Flat Source}

A flat-source approximation (radial and azimuthal) is the most conservative distribution to represent power distributions within a reactor with respect to the leakage of fast neutrons to the RPV. Since the outer assemblies contribute the most neutron fluence to the RPV and have an over-estimated neutron source in a flat source it is considered to be the most conservative in terms of lifetime assessment. Therefore, the results obtained from a flat source define the upper limit for the maximum damage at a given power level. The source is simply sampled in all fuel rods with equal probability using a U-235 fission spectrum. (Using U-235 spectrum is non-conservative, but its fluence-reducing effect is estimated to be several times smaller than the fluence-increasing effect of the spatially flat source.)

\subsection{Center-peaked Source}

In order to generate 3D fission source distributions the KENO-VI [13] sequence was used with a 238group library optimized for LWR eigenvalue calculations. KENO-VI models exact geometry via 3D MC (Monte Carlo). Radially, the model extends through the RPV and axially it extends through the fuel top 

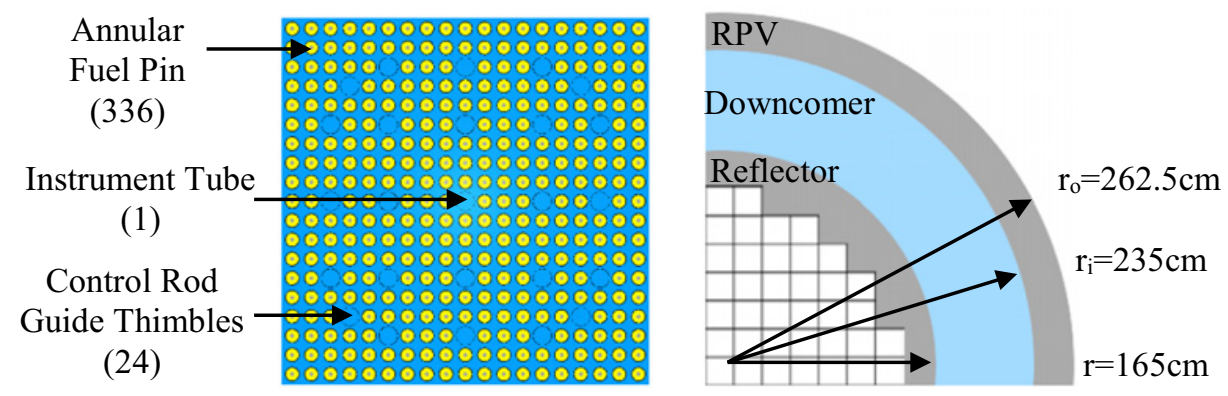

Figure 1. A $19 \times 19$ assembly and the radial core layout of half of the $I^{2} S-L W R$ through the RPV.

and bottom nozzles. Within the core, spacer grids are explicitly modelled. A typical fuel assembly is shown in Fig. 1. In order to accomplish reasonable results, first a problem-specific AMPX cross section library was generated taking into account the geometry of the annular fuel pins for multi-group selfshielding effects. Using MC to obtain a full-core spatial flux distribution requires many particles for spatial source convergence. Since the version of KENO-VI being used is serial, parallel computing was emulated by concurrently running 16 separate KENO-VI inputs with different random seeds. Each case simulated 60 million particles for a total of 960 million source particles. On average each run took a wallclock time of 10.7 hours. By running 16 simulations in parallel a total of more than 170 hours ( $>1$ week) of CPU-time was performed in 10.7 hours. The resulting fission distributions can then be averaged to reduce the uncertainty along with using quarter-core symmetry to further reduce uncertainty. Each pin is represented by 12 axial regions leading to a source represented with a $247 \times 247 \times 12$ spatial mesh and a 238-group energy structure. The reported relative uncertainty in $99 \%$ of voxels were converged within $2 \%$. With a uniform fuel distribution and no control rods or absorbers, the fission density is strongly center-peaked (radially and axially), more so than any realistic loading pattern, and thus presents a practical minimum-fluence bounding case. The radial source distribution with associated peaking can be seen in Fig. 2.

\section{Shielding Analysis with MAVRIC}

The MAVRIC sequence [15] implements a hybrid deterministic-stochastic approach to neutral-particle transport. The CADIS [16] methodology is used to bias particles toward a user-defined region and response of interest, here being DPA or fast fluence in the RPV. MAVRIC implements CADIS by first solving an adjoint $S_{N}$ (discrete ordinates) approximate representation of the problem on a Cartesian mesh, which is used to generate an importance map. The importance map is then used as weight windows for particle biasing in an MC simulation. Furthermore, MAVRIC can use the FW-CADIS (ForwardWeighted CADIS) [17] methodology which weights the adjoint source by the inverse of a forward $S_{N}$ solution in an attempt to create an importance map that biases particles equally toward tallies in different regions aimed at achieving near-uniform statistical uncertainties. For difficult shielding problems the CADIS and FW-CADIS methods have potential to reduce the computational time needed to achieve desired statistical convergence by orders of magnitude compared to analog MC.

For each fixed source, separate MAVRIC simulations were ran using the CADIS method to obtain fast neutron fluence $(>1 \mathrm{MeV}$ and $>0.1 \mathrm{MeV}$ ) and DPA rates within the RPV. Additional simulations used FW-CADIS to obtain tallies globally. The problem space used for the shielding calculation includes an air region outside of the RPV that extends to $300 \mathrm{~cm}$ radially. Axially, the model extends through the elevation corresponding to the assembly top and bottom nozzle over a range of $419 \mathrm{~cm}$. A uniform mesh of $120 \times 120 \times 96$ voxels was used in $S_{N}$ calculations. The forward flux and adjoint function distribution 


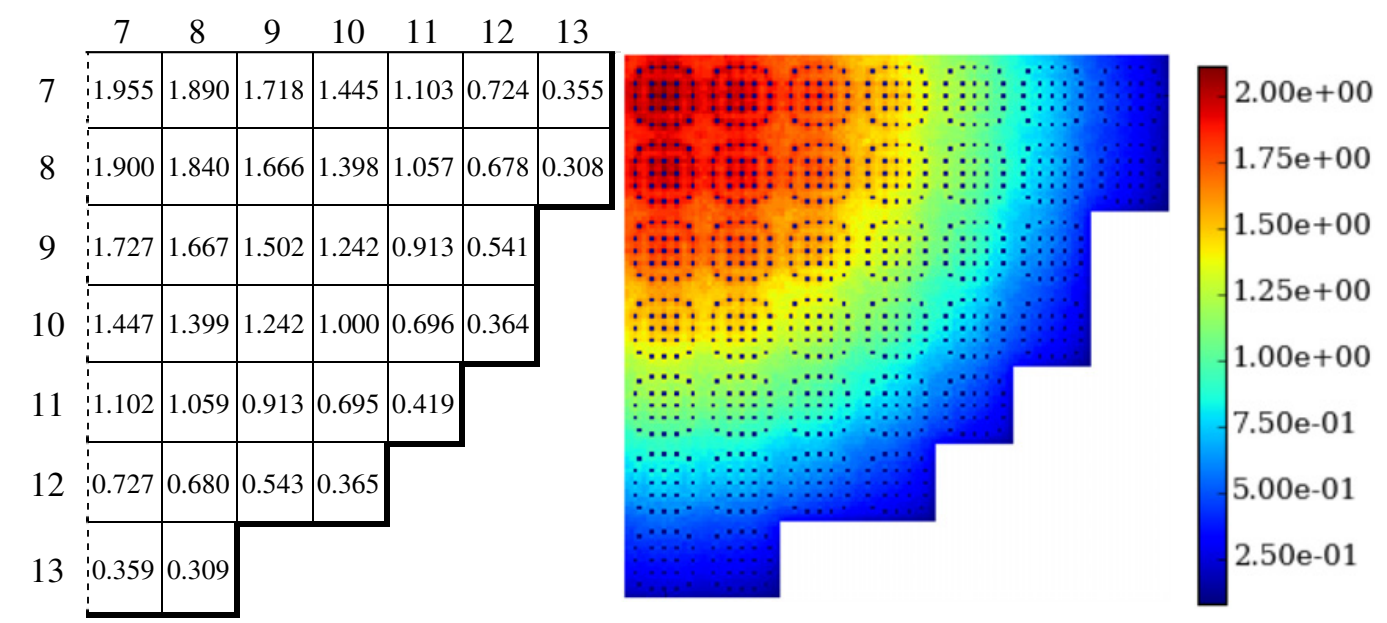

Figure 2. Assembly-averaged radial power profile for a center-peaked core entirely made of uniform fresh fuel.

were solved using a 27-group neutron library while the MC portion used a finer 200-group library. The latter 200-group library is optimized for shielding calculations in LWRs, which primarily is dependent on the streaming of fast neutrons. A total of 100 batches with 100,000 particles per batch were used in the MC portion. Both simulations (flat source and center-peaked source) took roughly the same amount of time since the problems are similar. The $\mathrm{S}_{\mathrm{N}}$ portion took $\sim 4.3 \mathrm{hrs}$ and the MC portion of simulation took $\sim 6.3$ hours for a total time of $\sim 10.6 \mathrm{hrs}$. Cartesian and cylindrical tallies were used to evaluate distributions of interest throughout the problem and the RPV.

\subsection{Fast Neutron Fluence Envelope}

As mentioned before, two limiting source distributions were implemented in order to obtain an envelope expected to bound the RPV neutron damage. The flat source provides the conservative overestimate, while the center-peaked distribution leads to an underestimate. Figures 3 and 4 depict the azimuthallyaveraged radial distribution of the fast neutron $(>1 \mathrm{MeV}$ and $>0.1 \mathrm{MeV}$ ) fluence rates at the elevation of the core midplane.

The flat-source distribution leads to a fast neutron fluence 9.3-12.2 times higher than that of the center-peaked source radially at the midplane of the RPV. The fast neutron $(>1 \mathrm{MeV})$ fluence rate for the flat source case is below typical PWR fluence rates by about 4 times. The actual value (based on a more accurately modelled source distribution) is expected to lie somewhere in between the two cases examined. Additionally, due to inelastic downscattering in steel, there is a slight increase in the fast neutron $(>0.1 \mathrm{MeV})$ fluence rate at the inner portion of the RPV as can be seen in Fig. 4.

\subsection{Azimuthal Fluence Distribution and Impact of Cooling Channels in the Reflector}

Cylindrical tallying was used to obtain azimuthal fluence distribution at the RPV inner surface. Additionally, the impact of the radial neutron reflector was examined. The reflector is made primarily of stainless steel. Due to its proximity to the core and resulting gamma heating, it will have to be designed with cooling channels. The heating analysis has not been completed at this point, and therefore channels could not be modelled in detail. Instead, in order to understand their impact on shielding performance, a parametric study was performed varying homogenized mixture of steel and water in the reflector in 


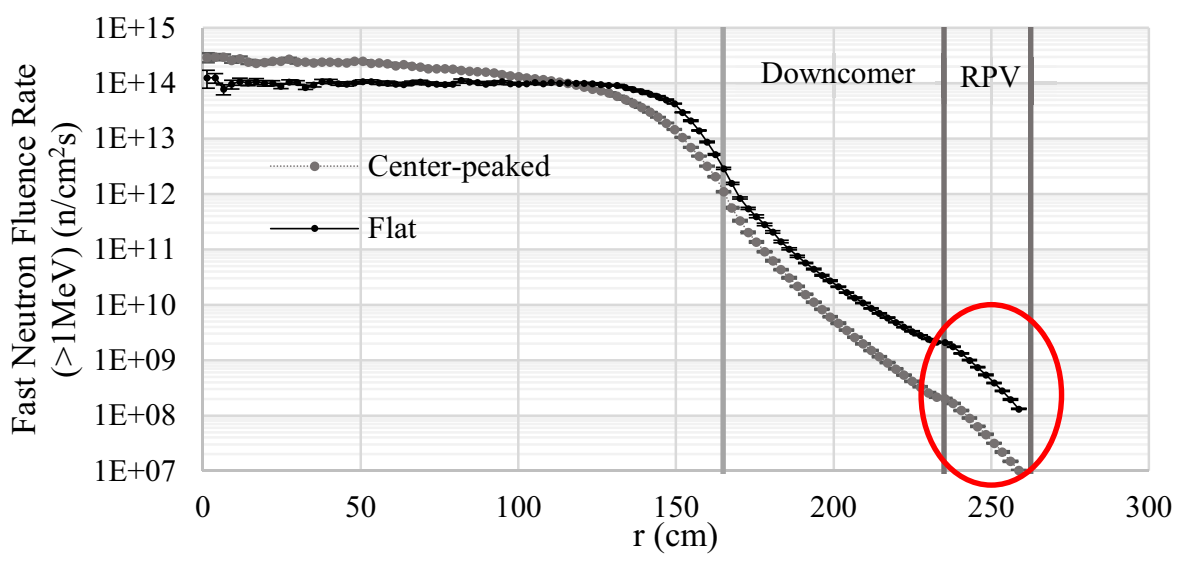

Figure 3. The azimuthally-averaged radial distribution of the fast neutron $(>1 \mathrm{MeV})$ fluence rates at the core midplane (with $\pm 1 \sigma$ uncertainty bars shown).

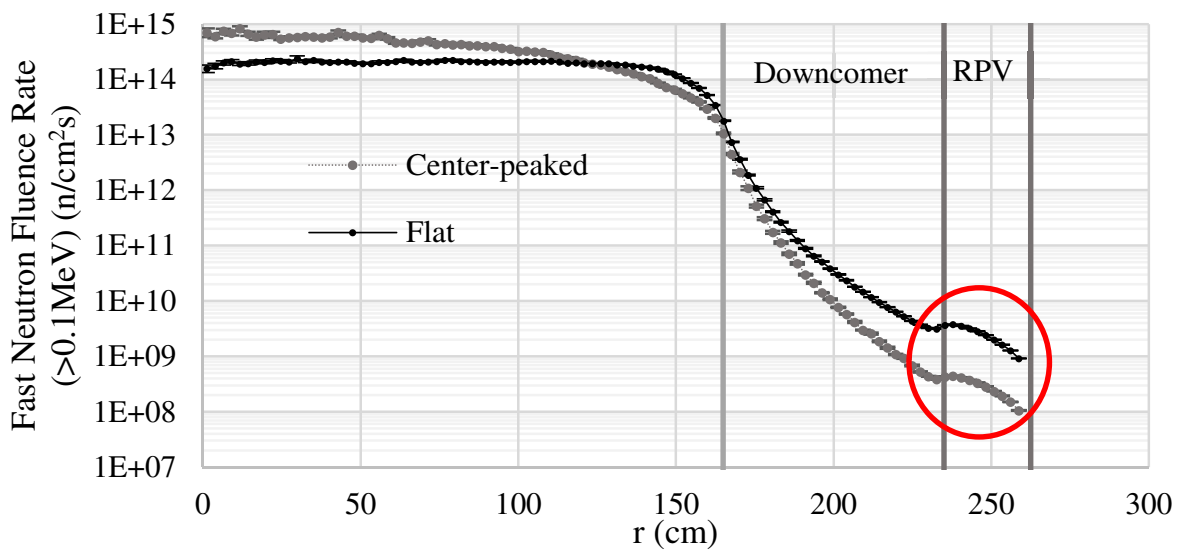

Figure 4. The azimuthally-averaged radial distribution of the fast neutron $(>0.1 \mathrm{MeV})$ fluence rates at the core midplane (with $\pm 1 \sigma$ uncertainty bars shown).

order to assess the change in fluence to the RPV. Cases considered are a $100 \% / 0 \%, 90 \% / 10 \%$, and $70 \% / 30 \%$ volume mixtures of steel and water respectively. Figure 5 shows the azimuthal distribution of the neutron flux around the core centreline at the RPV inner surface.

Compared to the pure steel case, assuming $10 \%$ water increases the fast fluence peak value at RPV inner surface by $10 \%$, and assuming $30 \%$ water increases the fluence peak value by $41 \%$. Table 1 shows accumulated fast fluence and DPA values for each case presented in this study.

\section{Conclusions}

Lifetime assessment of the radiation damage to the RPV in the proposed $\mathrm{I}^{2} \mathrm{~S}$-LWR integral configuration shows that levels are lower than that of a typical PWR even with an increased lifetime, as was expected. Major design changes affecting the neutron fluence are the use of a steel reflector and a larger downcomer region (decreasing the fluence rate) and a $40 \%$ higher power density and longer lifetime 


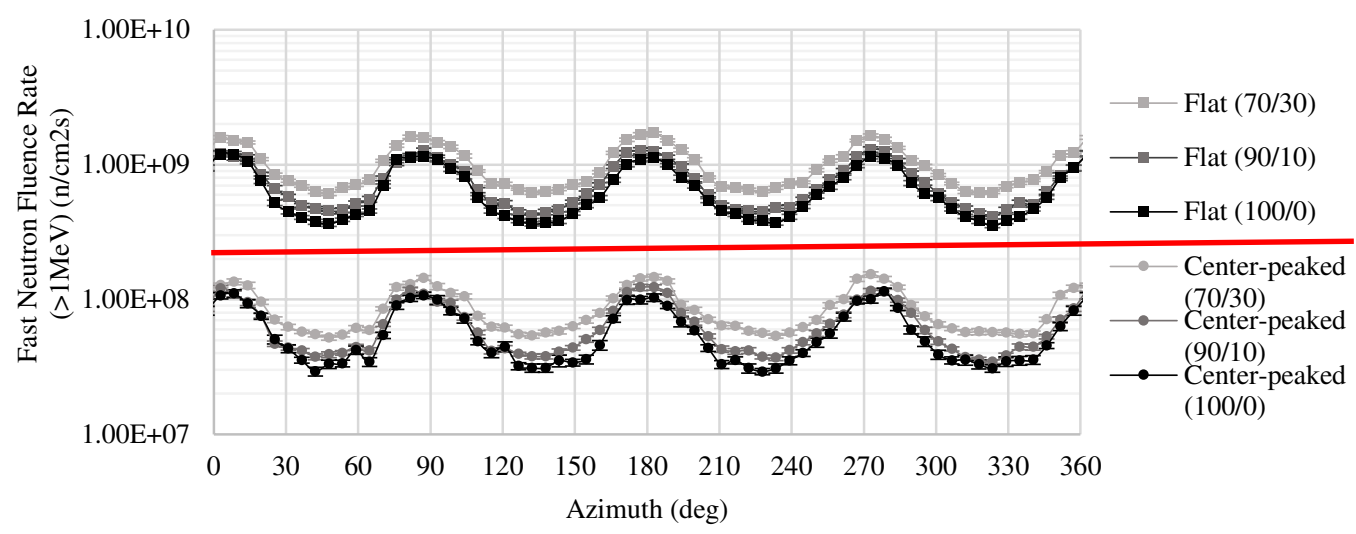

Figure 5. The fast neutron fluence rate $(>1 \mathrm{MeV})$ azimuthally at the core midplane at RPV inner surface for flat and center-peaked source representations and various reflector compositions.

Table 1. Accumulated fluence and DPA rates to the RPV.

\begin{tabular}{|c|c|c|c|c|c|c|c|}
\hline \multirow[t]{2}{*}{ Case } & \multicolumn{5}{|c|}{ 100-year Fast Fluence $(>1 \mathrm{MeV})\left[\left(10^{19}\right) \mathrm{n} / \mathrm{cm}^{2}\right]$} & \multirow[b]{2}{*}{$\mathrm{DPA} / \mathrm{s}$} & \multirow{2}{*}{$\begin{array}{l}\text { 100-year } \\
\text { DPA }\end{array}$} \\
\hline & Max** & Avg. 1/8T & Avg. 1/4T & Avg. 1/2T & Avg. 3/4T & & \\
\hline $\begin{array}{c}\text { Flat } \\
\left(70 / 30^{*}\right)\end{array}$ & $1.531 \pm 1 \%$ & $0.805 \pm 1 \%$ & $0.584 \pm 1 \%$ & $0.266 \pm 1 \%$ & $0.112 \pm 1 \%$ & $3.14 \mathrm{E}-12 \pm 2 \%$ & $8.93 \mathrm{E}-03 \pm 4 \%$ \\
\hline $\begin{array}{c}\text { Flat } \\
\left(90 / 10^{*}\right)\end{array}$ & $1.148 \pm 1 \%$ & $0.627 \pm 1 \%$ & $0.451 \pm 1 \%$ & $0.204 \pm 1 \%$ & $0.086 \pm 1 \%$ & $2.47 \mathrm{E}-12 \pm 2 \%$ & $7.01 \mathrm{E}-03 \pm 4 \%$ \\
\hline $\begin{array}{c}\text { Flat } \\
\left(100 / 0^{*}\right)\end{array}$ & $1.060 \pm 2 \%$ & $0.552 \pm 1 \%$ & $0.395 \pm 2 \%$ & $0.178 \pm 2 \%$ & $0.075 \pm 2 \%$ & $2.24 \mathrm{E}-12 \pm 4 \%$ & $6.38 \mathrm{E}-03 \pm 4 \%$ \\
\hline $\begin{array}{c}\text { Center-peaked } \\
(70 / 30 *)\end{array}$ & $0.143 \pm 2 \%$ & $0.076 \pm 2 \%$ & $0.052 \pm 2 \%$ & $0.021 \pm 2 \%$ & $0.008 \pm 2 \%$ & $4.08 \mathrm{E}-13 \pm 2 \%$ & $1.16 \mathrm{E}-03 \pm 2 \%$ \\
\hline $\begin{array}{c}\text { Center-peaked } \\
(90 / 10 *)\end{array}$ & $0.118 \pm 1 \%$ & $0.058 \pm 2 \%$ & $0.040 \pm 2 \%$ & $0.017 \pm 2 \%$ & $0.006 \pm 2 \%$ & $3.22 \mathrm{E}-13 \pm 2 \%$ & $9.14 \mathrm{E}-04 \pm 2 \%$ \\
\hline $\begin{array}{c}\text { Center-peaked } \\
\left(100 / 0^{*}\right)\end{array}$ & $0.113 \pm 3 \%$ & $0.054 \pm 2 \%$ & $0.037 \pm 2 \%$ & $0.015 \pm 3 \%$ & $0.006 \pm 4 \%$ & $2.87 \mathrm{E}-13 \pm 2 \%$ & $8.15 \mathrm{E}-04 \pm 2 \%$ \\
\hline
\end{tabular}

*The numbers represent ratios of homogenized steel-water volume percentages.

**Max and Avg. refer to azimuthal maximum and average values found at an elevation of the core midplane and radially in the inner portion of the RPV.

(increasing the fluence rate and fluence). The former effects exceed the latter, providing an additional margin compared to typical narrow-downcomer loop PWRs.

Fast fluence begins to be significant for welds with high $\mathrm{Cu}$ and $\mathrm{Ni}$ content around $2\left(10^{19}\right) \mathrm{n} / \mathrm{cm}^{2}$, which was the value considered conservatively to be the allowable upper limit. In general, RPV dosimetry assessment has many sources of errors coming from uncertainties in geometric models, crosssection libraries, solution techniques, fixed-source distributions, etc. [18-20]. The main uncertainty in this study was the source distribution since full temporal, spatial and energetic detail has not yet been implemented. To bound that uncertainty two fixed sources were used, creating an envelope on neutron damage to the RPV. The upper window from a high-leakage, flat source distribution resulted in a maximum 100 -year fast fluence $(>1 \mathrm{MeV})$ of $1.5\left(10^{19}\right) \mathrm{n} / \mathrm{cm}^{2}$ which is $75 \%$ of the $2\left(10^{19}\right) \mathrm{n} / \mathrm{cm}^{2}$ assumed upper limit. A low bound defined by a center-peaked source led to a maximum fast fluence of $1.4\left(10^{18}\right) \mathrm{n} / \mathrm{cm}^{2}$ which is 14 times below that limit. These results show that the increased risk of PTS from neutron fluence at RPV weld locations is not expected to be a consideration impacting or limiting core loading strategies. With respect to RPV dosimetry, no extra shielding material is currently needed in the $\mathrm{I}^{2} \mathrm{~S}$-LWR design process. However, changes due to other design aspects (i.e. reflector cooling channels) could alter results, but the current margin indicates that expected design changes will 
likely uphold acceptable levels. Future work will involve implementing an accurate representation of the spatial and temporal source distribution [21] in order to quantify different key radiation parameters as they accumulate in integral components within the pressure vessel throughout the lifetime of the $\mathrm{I}^{2} \mathrm{~S}-\mathrm{LWR}$.

\section{References}

[1] American Society for Testing and Materials, "Standard Guide for Characterizing Neutron Exposures in Iron and Low Alloy Steels in Terms of Displacements per Atom (DPA)", ASTM E693 (1994)

[2] B. Petrovic, "Integral Inherently Safe Light Water Reactor $\left(\mathrm{I}^{2} \mathrm{~S}-\mathrm{LWR}\right)$ Concept - Promoting Safety and Economics", Nuclear Engineering International, pp. 26-29 (March 2014)

[3] B. Petrovic, "Integral Inherently Safe Light Water Reactor ( $\left.{ }^{2} S-L W R\right)$ Concept: Extending SMR Safety Features to Large Power Output", Proceedings of ICAPP 2014 Charlotte, USA, Paper 14311 (April 6-9, 2014)

[4] B. Petrovic, K.W. Burn, M. Ciotti, L. Casalini, M. Sarotto, F. Franceschini, M. Carelli, J. Sejvar, J. Johnson, C. Lombardi, J. McKillop, D. Hartmangruber, "Design Features of the IRIS Reactor to Reduce Collective Dose During Operation and Maintenance and to Simplify D\&D", Proc. 8th Intl. Conf. on Nuclear Option in Countries with Small and Medium Electricity Grids, Dubrovnik, Croatia, paper S2-52 (May 16-20, 2010)

[5] American Society for Testing and Materials, "Standard Guide for Application of Neutron Transport Methods for Reactor Vessel Surveillance”, E706 (IID), ASTM E482-89 (1989)

[6] U.S. Nuclear Regulatory Commission, "Calculational and Dosimetry Methods for Determining Pressure Vessel Neutron Fluence”, Regulatory Guide 1.190 (2001)

[7] R.E. Maerker, M.L. Williams, and B.L. Broadhead, "Accounting for Changing Source Distribution in Light Water Reactor Surveillance Dosimetry Analysis", Nuclear Science and Engineering, Vol. 94 (1986)

[8] G.P. Cavanaugh et al., "Reduction in Reactor Vessel Irradiation Through Fuel Management", Transactions of the American Nuclear Society, Vol. 45, p. 98 (October 1983)

[9] M. Todosow et al., "Pressure Vessel Fluence Reduction Through Selective Fuel Assembly Replacement", Transactions of the American Nuclear Society, Vol. 45, p. 595 (October 1983)

[10] D. Cokinos et al., "Pressure Vessel Damage Fluence Reduction by Low-Leakage Fuel Management", Transactions of the American Nuclear Society, Vol. 45, p. 594 (October 1983)

[11] L.E. Steele, "Neutron Irradiation Embrittlement of Reactor Pressure Vessel Steels", IAEA Technical Report Series No. 163, Inernational Atomic Energy Agency, Vienna, Austria (1975)

[12] U.S. Nuclear Regulatory Commission, Radiation Embrittlement of Reactor Vessel Materials, Regulatory Guide 1.99 Revision 2 (1988)

[13] SCALE: A Modular Code System for Performing Standardized Computer Analyses for Licensing Evaluations, ORNL/TM-2005/39, Version 6.1, Vols. I-III (June 2011)

[14] Computing Radiation Dose to Reactor Pressure Vessel and Internals: State of the Art, report NEA/NSC/DOC(96)5, Nuclear Energy Agency, Organization for Economic Co-operation and Development (1997)

[15] D.E. Peplow, MAVRIC: Monaco with Automated Variance Reduction using Importance Calculations. ORNL/TM-2005/39. UT-Battelle, LLC. Oak Ridge National Laboratory (January 2009)

[16] J.C. Wagner and A. Haghighat, "Automated Variance Reduction of Monte Carlo Using Discrete Ordinates Adjoint Functions", Nuclear Science \& Engineering, Vol. 128, 186-208 (1998)

[17] J.C. Wagner, E.D. Blakeman, \& D.E. Peplow, "Forward-Weighted CADIS Method for Global Variance Reduction”, Transactions of the American Nuclear Society, Vol. 97. pp. 630-633 (2007) 


$$
15^{\text {th }} \text { ISRD }
$$

[18] M. Todosow and J.F. Carew, "Evaluation of Selected Approximations Used in Pressure Vessel Fluence Calculations", Transactions of the American Nuclear Society, Vol. 46, p. 658 (June 1984)

[19] B. Petrovic and A. Haghighat, "Effects of $S_{N}$ Method Numerics on Pressure Vessel Neutron Fluence Calculations," Nuclear Science and Engineering, Vol. 22, 167-193 (1996)

[20] A. Haghighat, M. Mahgerefteh, and B. Petrovic, "Evaluation of the Uncertainties in the Source Distribution for Pressure Vessel Neutron Fluence Calculations", Nuclear Technology, Vol. 109, 54-75 (January 1995)

[21] F. Franceschini, B. Petrovic, "Impact of the Detailed Fission Source Distribution on IRIS Shielding Analyses”, Nuclear Technology, Vol. 168, 431-437 (2009) 